\title{
Success of Endoscopy with Narrow Band Imaging in Diagnosis of Cervixcal Metaplasia
}

\author{
Salwa Samir Anter* \\ Department of Obstetrics Gynaecology, Egypt
}

*Corresponding author: Salwa samir Anter, Department of Obstetrics Gynaecology, Egypt.

Received Date: December 15, 2018

Published Date: January 02, 2019

\section{Abstract}

Background: Flexible magnifying endoscopy with narrow band imaging (ME-NBI) has outstanding diagnostic correctness for gastrointestinal Metaplasia and is hope for to be highly useful for diagnosis cervixcal Metaplasia in this study aiming to detect the feature findings and access the diagnostic power of ME-NBI for diagnosis of cervixcal Metaplasia.

Method: 20 women were undergoing vaginal smear and at the same time Flexible NBI-ME was performed. after. Written consent was obtained from patient's vaginal examination were done. Cusco speculum is used to examination a cervix by endoscopy using white light imaging, and. Narrow band image at long, middle, and short distances. Images and video of ME-NBI were taken to investigate the cervical lesions. The images were analysis built on cytology result (Figure 1).

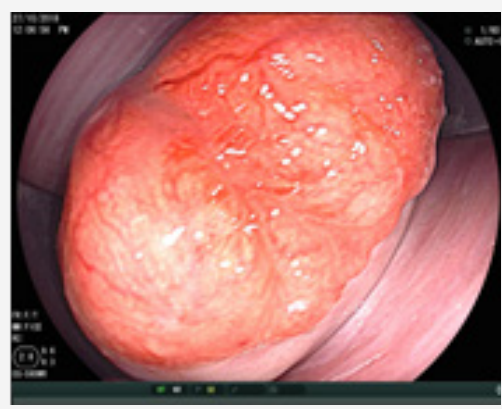

Figure 1: Vascular of cervix by light by blue band.

Keywords: Cervical; Metaplasia; Magnifying; Endoscopy; Narrow band; Imaging

Results

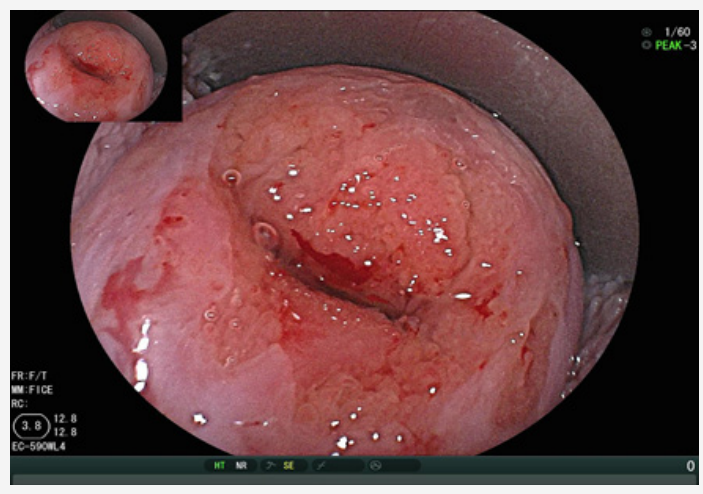

Figure 2: Metaplasia with narrow band image.
The NBI-ME images revealed the following abnormal findings: Tongue-like projections of the epithelium, Nabothian follicles, gland ostium, Metaplastic cells with special features (Figure 2-6).

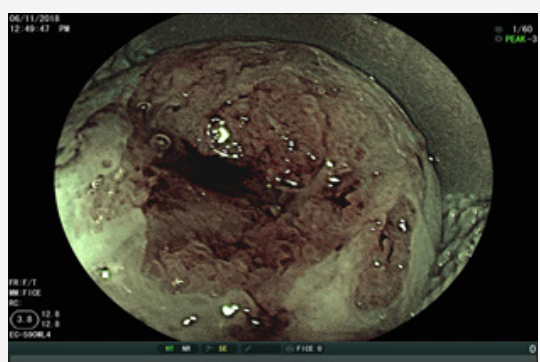

Figure 3: Metaplasia with narrow band image. 


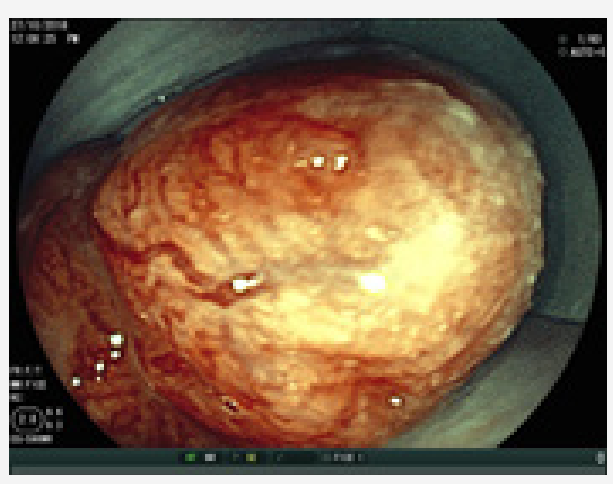

Figure 4: Vascular of cervix by green band.

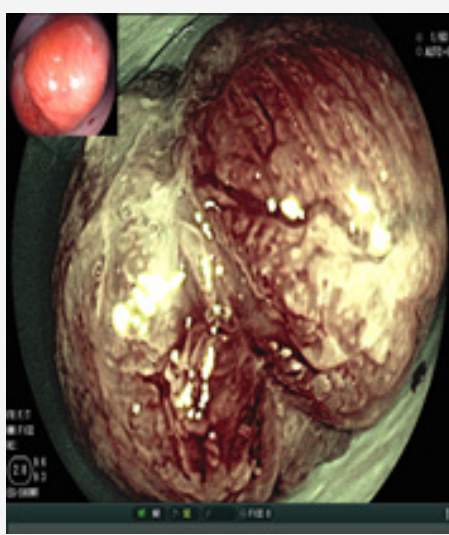

Figure 5: Metaplsia zona with transformation zon.

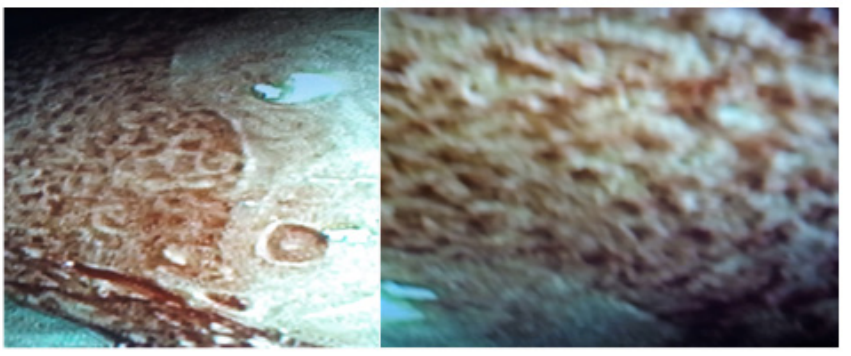

Figure 6: Metaplasia cells.

Transformation zone of type normal vascular of cervix and its change shape of all type of epithelium cells, cell nuclear density, thickness of epithelium. If you know how to diagnosis squamous Metaplasia 50 percent of correct diagnosis of cervical lesion Will be able to diagnosis.

\section{Conclusion}

This study indicates that ME-NBI may have novel value for Metaplasia diagnosis without use of acetoacetic acid or Lugol's iodine.

\section{Acknowledgement}

None.

\section{Conflict of Interest}

No conflict of interest. 